\title{
ON-ARRIVAL PLANNING FOR SUB-GRAPH ROUTING PROTECTION IN WDM NETWORKS
}

\author{
Darli A. A. Mello, Marcio S. Savasini, \\ Jefferson U. Pelegrini and Helio Waldman ${ }^{1}$ \\ ${ }^{1}$ Optical Networking Laboratory, State University of Campinas - UNICAMP \\ Av. Albert Einstein, 400 CP 6101, CEP 13083-970, Campinas - SP - Brazil \\ e-mail $\{$ darli $\}\{$ savasini $\}\{$ jpelegri $\}\{$ waldman $\} @$ decom.fee.unicamp.br
}

\begin{abstract}
:
In this paper we incorporate the rerouting of backup paths to the Call Admission Control algorithm of Sub-Graph Routing Protection (SGRP). Sub-Graph Routing Protection with On-Arrival Planning (SGRP-OAP) has the two-fold benefit of strongly reducing the blocking probability of the protected system while eliminating the physical rerouting of established connections, which is a major drawback of the original proposal of SGRP. The new scheme is so capacity-efficient that, for all investigated topologies, at low traffic intensity the blocking probability of the system protected against single link failures is the same as the blocking probability of the unprotected system. This is possible because the new protection scheme is extremely effective in using the idle network capacity to provide backup paths.
\end{abstract}

\section{INTRODUCTION}

Protection and Restoration are critical issues in the design of wavelength routed WDM networks [1]. Schemes which are efficient in terms of scalability, dynamicity, class of service, restoration speed and network utilization have been extensively researched. In wavelength routed networks traffic can be either static or dynamic. With static traffic connection requests are available all at once, and the path protection problem can be solved by Integer Linear Programming [2]. Conversely, with dynamic traffic connection requests arrive sequentially and exist for a finite duration, which demands heuristic methods for solving the path protection problem [3]. In this paper we consider only dynamic traffic. 
Frederick and Somani [4] have recently introduced Sub-Graph Routing Protection (SGRP), a path protection scheme suitable for dynamic traffic that exhibits improved capacity-efficiency, when compared to the already consolidated Backup Multiplexing [3]. However, the original publication on SGRP also highlighted one of its major drawbacks: upon occurrence of a link failure, even connections that do not traverse the faulty link may have to change their path or wavelength to accommodate others, causing inconvenient service interruptions. We call this altruistic reassignment, to distinguish it from the regular reassignment that a connection has to undergo when a link that it traverses fails. Altruistic reassignment is a cumbersome maneuver that requires care in order not to disturb costumers that are not directly harmed by the failure.

Initially conceived to support single link failures, SGRP has been also extended to support multiple, node and Shared-Risk Link Group failures [5]. In the same paper the problem involving altruistic reassignment was readdressed by imposing constraints on the RWA algorithm that suppress altruistic path or wavelength reassignment. It has been observed that if altruistic path reassignment is suppressed, but altruistic wavelength reassignment allowed, the blocking probability is slightly reduced for some network topologies, but the overall reassignment probability stays above $90 \%$. Since the reduction in blocking probability is very slight, we will compare our results with the blocking of the original proposal, which is about the same as for the path constrained case. If both path and wavelength altruistic reassignments are suppressed, the blocking probability increases significantly.

We have recently proposed an inter-arrival planning of backup paths [6] that reduces altruistic reassignment while preserving the blocking probability. We exploited the fact that it is cheaper to recalculate backup paths than to perform altruistic reassignment, since the former does not deal with reconfiguration of connections physically established in the network, but only with logical connections stored in the system. Sub-Graph Routing Protection with Inter-Arrival Planning does not modify the Call Admission Control algorithm, exhibiting therefore blocking probabilities similar to the original proposal, and reducing the altruistic reassignment probability, but not completely eliminating it.

In this paper we present the Sub-Graph Routing Protection with On-Arrival Planning scheme (SGRP-OAP), an evolution that exhibits extremely high capacity efficiency at the expense of a moderate computational cost. Contrasting with Sub-Graph Routing Protection with Inter-Arrival Planning, SGRP-OAP modifies the Call Admission Control algorithm of the original proposal, exhibiting blocking probabilities considerably lower than the original proposal while completely eliminating altruistic reassignment, being therefore recommendable when provisioning time is compatible with the processing time of the Call Admission Control algorithm. In this paper we simulate only single link failures, but future work could extrapolate the developed concepts to other 


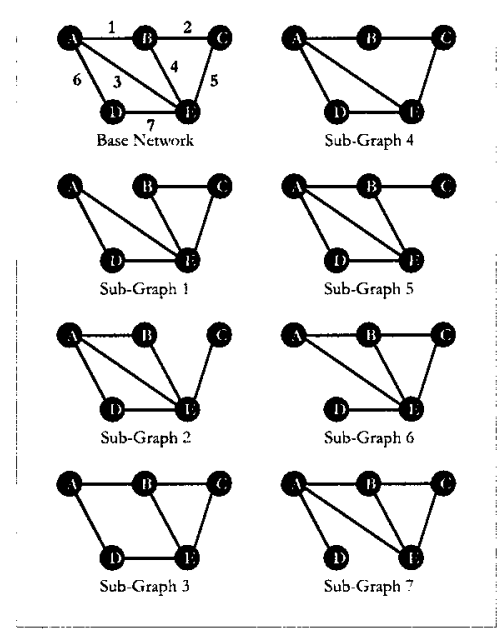

Figure 1. Sub-Graph Routing Protection

scenarios. The remainder of this paper is divided into five sections. Section 2 explains the original proposal of Sub-Graph Routing Protection. In Section 3 the Sub-Graph Routing Protection with On-Arrival Planning scheme is developed. Section 4 presents the methods used to evaluate the performance of the new scheme. Section 5 shows the simulation results, and Section 6 concludes the paper.

\section{SUB-GRAPH ROUTING PROTECTION}

The main idea of Sub-Graph Routing Protection is rather clever. A network topology can be represented by an undirected graph $G(V, E)$ with a vertex set $V$ and an edge set $E$. The set $V$ represents nodes and the set $E$ represents bidirectional links. $G(V, E)$ is called the base network. A single failure of edge $e_{i}$ can be represented by sub-graph $G_{i}=G-e_{i}$ : the original graph $G$ without edge $e_{i}$. In this way all possible single link failures in the network can be represented by $L$ sub-graphs, where $L$ is the cardinality of the edge set $E$, as depicted in Figure 1. In Sub-Graph Routing Protection, a connection request is only accepted if it can be successfully routed in each of the $L$ sub-graphs and in the base network. In case of a link failure, the network immediately incorporates the state represented by the corresponding sub-graph.

In the strategy proposed by the first paper on Sub-Graph Routing Protection [4] the RWA of connections in the sub-graphs is independent of the base network configuration. In the example of Figure 2, the connection path in the base network and sub-graphs is the shortest path between source and destina- 


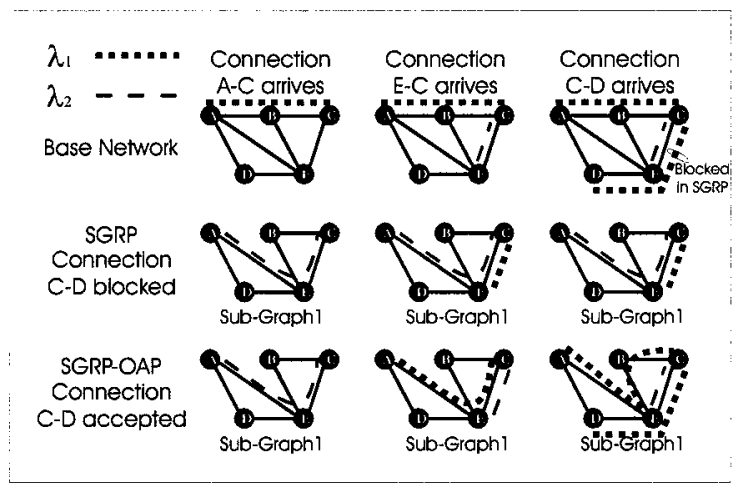

Figure 2. SGRP and SGRP-OAP in Sub-Graph 1

tion nodes, and wavelength is randomly chosen. Note that connection E-C uses wavelength 2 in the base network, and wavelength 1 in sub-graph 1 . If a failure occurs on link 1, connection E-C undergoes an altruistic reassignment.

\section{SUB-GRAPH ROUTING PROTECTION WITH ON-ARRIVAL PLANNING}

In this section we introduce the On-Arrival Planning Strategy for Sub-Graph Routing Protection (SGRP-OAP). Here we modify the Call Admission Control algorithm of the original proposal to reroute backup routes stored in sub-graphs to better adjust the new incoming request. The algorithm is applied after the arrival of each connection request:

\section{In the Base Network:}

1. The connection is routed in the physically shortest path;

2. If this is not possible, the connection is blocked.

\section{In each Sub-Graph $G_{i}$ :}

1. Initial state of $G_{i}$ is saved;

2. All connections are cleared from $G_{i}$;

3. Two connection lists are generated, including the new connection request: $C_{F}$ - connections that traverse link $i$ in the base network, $C_{N}-$ connections that do not traverse link $i$ in the base network;

4. Connections of list $C_{N}$ are routed in $G_{i}$ as in the base network;

5. Connections of list $C_{F}$ are routed using the shortest path among all wavelength planes, in order of arrival;

6. If it is not possible to route all connections, the connection request is blocked and the initial states of the sub-graphs are restored. 
Upon the arrival of a connection request, SGRP-OAP "copies" in all subgraphs the base network configuration of connections which do not traverse the missing link of each sub-graph in the base network. The remaining connections, which would anyway be rerouted, are then routed within the residual capacity using the shortest path among all wavelength planes. In this way altruistic reassignment, an important concern in previous works on SGRP, is eliminated. In the example of Figure 2, when connection E-C arrives, it is included in list $C_{N}$ of sub-graph 1 , whereas connection A-C is included in list $C_{F}$, since A-C traverses link 1 in the base network. Connection E-C is routed first, succeeded by connection A-C, according to steps 4 and 5 of the SGRPOAP algorithm. When connection C-D arrives, it is also included in list $C_{N}$ of sub-graph 1. So, after connections of list $C_{N}$ (E-C and C-D) are routed in sub-graph 1, connection A-C has to be again rerouted in the shortest path among the two wavelength planes, which is 3 hops long. Note that the final configuration is free of altruistic reassignment.

\section{PERFORMANCE EVALUATION}

We evaluate the performance of SGRP-OAP through simulations performed by the optical networks simulator developed at the OptiNet, Optical Networking Laboratory at the State University of Campinas - UNICAMP. The simulator was written in the Java programming language. Uniform traffic is assumed. The arrival of connection requests follows a Poisson distribution, and the holding time is exponentially distributed. We simulated three network topologies [5]: the 14-node, 23-link NSFNet; 9-node, 18-link 3x3 Mesh-Torus; and the 11-node, 22-link NJLATA. All links are bidirectional with 16 wavelengths. The curves are calculated by averaging the results of the 10 last rounds of a series of 11 , each with 1000 connection requests, to simulate a steady state network occupancy.

In previous papers on SGRP mainly two performance metrics have been investigated: blocking probability and reassignment probability. The reassignment probability is the probability that a connection has to be rerouted upon the occurrence of a single link failure anywhere in the network. The reassignment probability consists of two components. The first component is the regular reassignment that a connection has to undergo when a link that it traverses fails, which depends solely on the average connection length and is close to the reassignment probability observed in Backup Multiplexing. The second component is the altruistic reassignment, which is absent when Backup Multiplexing is used. Since in SGRP-OAP no altruistic reassignment is allowed, the reassignment probability strongly decreases when compared to the original proposal, and only the regular reassignment remains. Therefore when SGRP-OAP is used the assessment of the reassignment probability metric is no 
longer important, and only the blocking probability is investigated. The performance of SGRP-OAP is compared with the Unconstrained RWA case because the latter possesses equal or lower blocking performance when compared to previously published RWA policies.

\section{RWA in the Base Network}

Connections are routed in the base network using Dijkstra's shortest path algorithm, in terms of hop count, applied to the physical network topology. If there are more than one shortest path, one is chosen randomly. Wavelength selection follows the Random Fit scheme.

\section{RWA in Sub-Graphs}

Unconstrained $R W A$ : refers to the seminal paper on sub-graph routing [4], which in sub-graphs used the same RWA scheme as in the base network.

Sub-Graph Routing Protection with On-Arrival Planning: connection requests are accepted or blocked according to the steps described in Section 3. The RWA of connections in list $C_{F}$, step " 5 " of the SGRP-OAP algorithm, chooses the wavelength with the shortest path, which is determined by applying Dijkstra's shortest path algorithm to all wavelength planes.

We have investigated two metrics to assess the performance of SGRP-OAP: the total blocking probability, and the base network blocking probability. For a connection to be accepted, it must be successfully routed first in the base network, and then in the $L$ sub-graphs. The total blocking probability is the probability of a connection request being blocked because it could not be routed in the base network or subgraphs. The base network blocking probability is the probability of a connection request being blocked because it could not be routed in the base network. It reflects the occupancy of the network without protection, since the allocation of protection resources is related to the routing of connections in sub-graphs.

\section{SIMULATION RESULTS AND DISCUSSION}

The three simulated network topologies exhibit analogous behaviors, but some effects are more pronounced in certain topologies. The results for the $3 \times 3$ Mesh Torus can be found in Figure 3. Here the benefits of SGRP-OAP in comparison with the original proposal are explicit. A remarkable result is that the total blocking probability and the base network blocking probability curves only diverge considerably after a load of 10 Erlangs per node, at approximately $1 \%$ blocking probability. This means that for blocking probabilities lower than $1 \%$, protection can be implemented at almost zero cost in terms of capacity. The gains in using SGRP-OAP for the NJLATA topology are still considerable, but less pronounced than for the $3 \times 3$ Mesh Torus Topology, as depicted in Figure 4. Again the blocking probability is considerably less than for the Unconstrained RWA case. The total blocking probability and the base network 
blocking probability curves diverge after 5 Erlangs per node, at approximately $2 \%$ blocking probability. The NSFNet topology exhibits the lowest gains when using SGRP-OAP, as shown in Figure 5. The total blocking probability and the base network blocking probability curves diverge after a load of approximately 3 Erlangs per node at near-zero blocking probability.
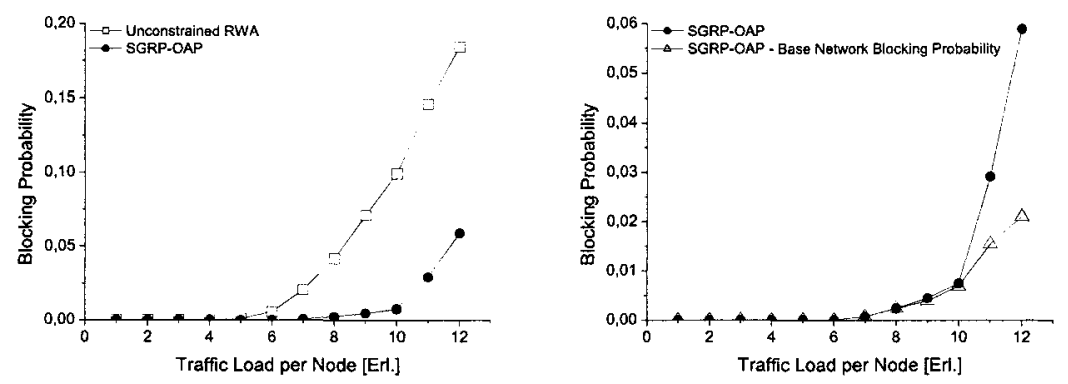

Figure 3. $3 \times 3$ Mesh Torus
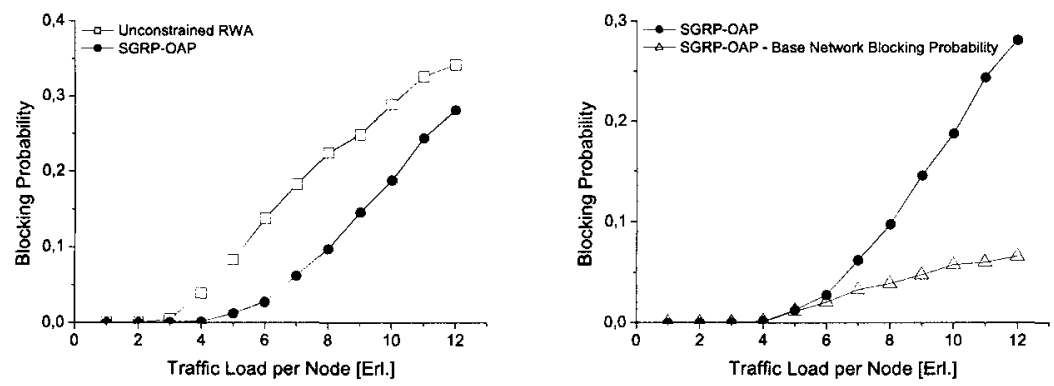

Figure 4. NJLATA
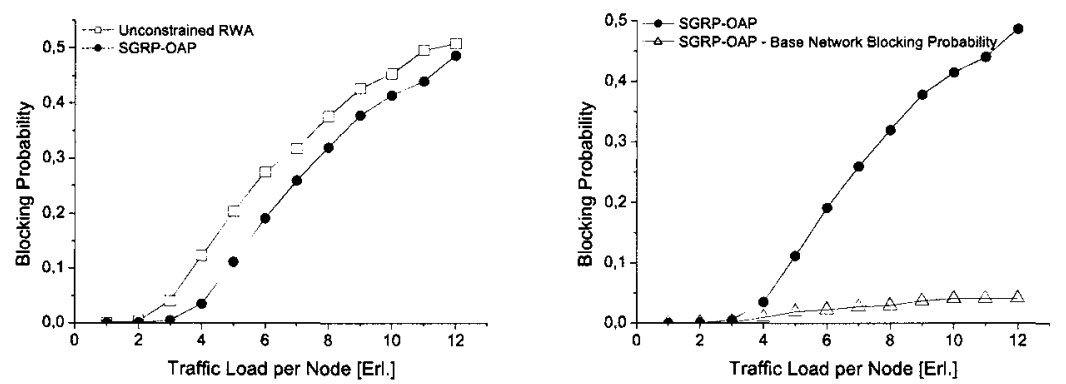

Figure 5. NSFNet

Concerning computational complexity, SGRP-OAP is fairly implementable if we consider current network dinamicity. Intensive computational time is only needed for provisioning a new connection, when Dijkstra's shortest path algorithm, with worst case complexity $O\left(N^{2}\right)$, is applied to W wavelength planes for source-destination pairs of connections which are subject to rerout- 
ing in case of a link failure. Therefore the provisioning time scales with $\mathrm{W}$, L (number of sub-graphs), the network occupancy and the complexity of the shortest path algorithm.

\section{CONCLUSION}

Sub-Graph Routing Protection has been recently introduced as a capacityefficient path protection scheme. The rerouting of backup paths in the call admission control algorithm boosts capacity-efficiency of the original proposal while completely eliminating altruistic reassignment. For the three simulated network topologies, Sub-Graph Routing Protection with On-Arrival Planning exhibited, at low traffic intensity, a blocking probability for the system protected against single link failures that is the same as for the unprotected system, which means that for this region SGRP-OAP is optimal in terms of capacityefficiency. For higher blocking probabilities, there is still no tight lower bound to support an almost-optimality claim for SGRP-OAP, although its extraordinary performance suggests that this can be true. There is still much to be researched on SGRP-OAP, and future work can concentrate on network management, failure isolation, restoration speed, and finding how close SGRP-OAP is from some tighter lower bound on the blocking probability for a protected network. Besides, the performance of SGRP-OAP in multiple-failure scenarios has to be also investigated. Finally, SGRP-OAP is a strongly recommended path protection scheme in scenarios where capacity-efficiency is required.

\section{ACKNOWLEDGMENT}

This work has been supported by FAPESP, CNPq and Ericsson Telecom. S.A., Brazil.

\section{REFERENCES}

[1] R. Ramaswami and K. N. Sivarajan, Optical Networks: a Practical Perspective. Morgan Kaufmann Publishers, 2002.

[2] S. Ramamurthy and B. Mukherjee, "Survivable WDM mesh networks, part I - protection," in Proc. IEEE INFOCOM'99, pp. 744-751, 1999.

[3] G. Mohan, C. S. R. Murthy, and A. K. Somani, "Efficient algorithms for routing dependable connections in WDM optical networks," vol. 9, no. 5, pp. 553-566, 2001.

[4] M. T. Frederick and A. K. Somani, "A single-fault recovery strategy for optical networks using subgraph routing," in Proc. ONDM'03, pp. 549-568, Feb. 2003.

[5] P. Datta, M. T. Frederick, and A. K. Somani, "Sub-graph routing: a novel fault-tolerant architecture for shared-risk link group failures in WDM optical networks," in Proc. $D R C N^{\prime} 03$, Oct. 2003.

[6] D. A. A. Mello, J. U. Pelegrini, M. S. Savasini, G. S. Pavani, and H. Waldman, "Interarrival planning for sub-graph routing protection in WDM networks," in Proc. ICT'04, Aug. 2004. 